\section{Monitoring der Schachtanlage Asse II}

\author{
von Urban Regenauer und Christiane \\ Wittwer, Bundesamt für Strahlenschutz
}

Die Schachtanlage Asse II ist ein ehemaliges Salzbergwerk nahe Wolfenbüttel in Niedersachsen. Von 1967 bis 1978 wurden 125.787 Gebinde mit schwach- und mittelradioaktiven Abfällen in das Bergwerk eingelagert. Seit 1988 dringen steinsalzgesättigte Zutrittslösungen aus dem Nebengebirge in das Bergwerk ein. Zur Überwachung der durch abgeführte Grubenluft möglicherweise in die Umgebung austretenden Radionuklide wurde ein umfangreiches Monitoring-Konzept eingeführt. Ziel dieses Artikels ist, die aktuelle Situation in der Schachtanlage Asse II mit besonderem Fokus auf dieses Monitoring-Konzept zu erläutern. Dies geschieht basierend auf einem Überblick über die Geschichte der Asse, die ursprünglich als Forschungsbergwerk diente. Weiterhin wird der regionale Begleitprozess vorgestellt, der 2007 ins Leben gerufen wurde.

\section{Einführung}

Die Schachtanlage Asse II ist ein ehemaliges Salzbergwerk nahe bei Wolfenbüttel in Niedersachsen. Von 1967 bis 1978 wurden 125.787 Gebinde mit schwach- und mittelradioaktiven Abfällen in das Bergwerk eingelagert. Seit 1988 dringen steinsalzgesättigte Zutrittslösungen aus dem Nebengebirge in das Bergwerk ein. Durch den Gebirgsdruck auf die zahlreichen dicht beieinander liegende Abbaue in der Südflanke folgen Gebirgsbewegungen, die bereits zu einer Schädigung des Salzgesteins und des Nebengebirges geführt haben. Da die Schädigungsprozesse andauern, besteht die Gefahr, dass der Lösungszutritt auf ein unbeherrschbares Niveau ansteigt und die Schachtanlage absäuft. Daher ist es notwendig, die Schachtanlage Asse II so schnell wie möglich sicher stillzulegen. Nach einem im Laufe des Jahres 2009 durchgeführten Vergleich verschiedener Stilllegungsoptionen verfolgt das BfS das Ziel, die radioaktiven Abfälle aus der Schachtanlage Asse II zu bergen und nach Konditionierung und Zwischenlagerung in ein anderes noch zu bestimmendes Endlager zu bringen.
Der derzeit laufende Entscheidungsprozess zur Stilllegung und Rückholung der Abfälle wird von der Asse-II-Begleitgruppe des Landkreises Wolfenbüttel aus örtlichen Stakeholdern und Bürgerinitiativen begleitet. Diese gibt ihre Empfehlungen und Erwartungen $u$. a. auch zum Monitoring an den Betreiber weiter.

Die eingelagerten Abfälle setzen flüchtige radioaktive Stoffe frei, die in die mit Grubenluft versorgten Grubenbereiche gelangen und mit der abgeführten Grubenluft (Abwetter) in die Umgebung abgeleitet werden. Emission und Immission von radioaktiven Stoffen werden messtechnisch überwacht. Als vertrauensbildende Maßnahmen werden mehr Messungen als die fachlich und gesetzlich erforderlichen durchgeführt. Das Messprogramm und die durch die abgeleiteten radioaktiven Stoffe verursachte potenzielle Strahlenexposition werden im Hinblick auf den Schutz der Bevölkerung und die Umwelt bewertet.

\section{Hintergrund}

In der etwa acht Kilometer östlich von Wolfenbüttel gelegenen Schachtanlage Asse II wurden von 1906 bis 1963 Stein- und Kalisalze gewonnen. 1965 übernahm die Gesellschaft für Strahlen- und Umweltforschung mbH München (heute: Helmholtz Zentrum München - Deutsches Forschungszentrum für Gesundheit und Umwelt - HMGU) im Auftrag des Ministeriums für Bildung und Forschung (BMFT heute BMBF) die Schachtanlage Asse II, um radioaktive Abfälle einzulagern und zu forschen. Für den Betrieb der Schachtanlage Asse II wurde durch die Gesellschaft für Strahlen- und Umweltforschung $\mathrm{mbH}$ ein Institut für Tieflagerung (IfT) gegründet. In der Zeit von April 1967 bis Dezember 1978 wurden in der Schachtanlage Asse II 124.494 Gebinde mit schwachradioaktiven Abfällen und 1.293 Gebinde mit mittelradioaktiven Abfällen eingelagert. Die Abfälle lagern überwiegend in elf Kammern auf der 750-m-Sohle und in jeweils einer Kammer auf der 725-m- und 511-m-Sohle. Die Einlagerung der radioaktiven Abfälle erfolgte seinerzeit ohne vorherige Festlegung eines Stilllegungskonzeptes. Dies entspricht nicht den heutigen atomrechtlichen Vorgaben, die ein Stilllegungskonzept und einen Langzeitsicher- 
heitsnachweis für eine Planfeststellung voraussetzen (AtG 2011).

Zur Stilllegung der unter Bergrecht als Forschungsbergwerk betriebenen Schachtanlage wurde 2007 ein Abschlussbetriebsplan vorgelegt (Schauermann 2007). Dieser sah eine weitgehende Verfüllung des Bergwerkes überwiegend mit Beton und Schotter sowie eine anschließende Flutung des nicht erreichbaren Hohlraums mit technisch definierter Lösung (sog. Schutzfluid) vor.

Insbesondere aus dem Kreis der Bürgerinitiativen wurde bereits 2007 gefordert, die Schachtanlage unter Atomrecht zu stellen, da dieses im Unterschied zum Bergrecht ein obligatorisches Planfeststellungsverfahren mit Öffentlichkeitsbeteiligung vorsieht. Ein entsprechendes Klageverfahren einer Anwohnerin war anhängig. Im Sommer 2008 wurde erstmals öffentlich bekannt, dass radioaktiv kontaminierte Lösung in nicht genutzte Hohlräume gepumpt wurde, die auf tieferen Sohlen lagen.

In einem Ministergespräch im Herbst 2008 (am 4.9.2008) zwischen Bundesminister Gabriel (BMU), Bundesministerin Schavan (BMBF) und dem niedersächsischen Umweltminister Sander wurde Einigung dahingehend erzielt, dass die Stilllegung nach Atomrecht und ein Betreiberwechsel von HMGU auf das Bundesamt für Strahlenschutz (BfS) erfolgen sollen. Der Kabinettsbeschluss hierzu wurde daraufhin am 5.11.2008 gefällt. Der Betreiberwechsel vom HMGU auf das BfS erfolgte zum 1.1.2009.

\section{Der Begleitprozess Asse}

Die ab dem Jahr 2007 zunehmende Kritik an dem ehemaligen Betreiber HMGU betraf insbesondere das Stilllegungskonzept und die aus Sicht der Kritiker mangelnde Informationspolitik und die eingeschränkte Transparenz des ehemaligen Betreibers.

Im November 2007 wurde zwischen dem niedersächsischen Ministerium für Umwelt, Energie und Klimaschutz (NMU), dem Bundesministerium für Umwelt, Naturschutz und Reaktorsicherheit (BMU) und dem BMBF eine interministerielle Vereinbarung getroffen, dass für die Stilllegung der Schachtanlage Asse II ein Begleitprozess mit Beteiligung der örtlichen Stakeholder zu initiieren sei. Es wurde der Auftrag formuliert, eine Begleitgruppe zu bilden, die Empfehlungen ausspricht. Hiermit wurde entschieden, eine Bürgerbeteiligung an einem Großprojekt bereits in der Entwurfsphase und weit vor dem eigentlichen Planfeststellungsverfahren einzurichten.

Die Konstituierung der Begleitgruppe AsseII (,A2B“) erfolgte am 18.1.2008. An dem vom Landrat geführten Gremium sind der Landkreis Wolfenbüttel, Abgeordnete aller Kreistagsparteien, die örtlichen Bürgermeister und Vertreter der Bürgerinitiativen als stimmberechtigte Mitglieder beteiligt. Das BfS als Betreiber, das BMU als Aufsichtsbehörde über das BfS und Bundesaufsicht über das NMU sowie das NMU als atomrechtliche Aufsicht und Genehmigungsbehörde sind als informelle Teilnehmer zeitweise vertreten.

Zur fachlichen Beratung der A2B wurde eine „Arbeitsgruppe Optionenvergleich“ (AGO) eingerichtet, deren Konstituierung am 5.3.2008 erfolgte. Das Gremium hat sich mittlerweile in „Arbeitsgruppe Optionen-Rückholung“ umbenannt. An der AGO wurden drei von der A2B ausgewählte Experten sowie das BfS und der Projektträger Wassertechnologie und Entsorgung/Karlsruhe (PTKA-WTE; heute Karlsruher Institut für Technologie, KIT) beteiligt. Bis zur Betriebsübernahme lag die Federführung der AGO beim BfS und dem KIT (früher PTKA-WTE). Das Zusammenspiel der Begleitgruppe Asse-II und der Arbeitsgruppe Optionen-Rückholung wird in der folgenden Abbildung graphisch dargestellt (s. Abb. 1).

Abb. 1: Zusammenspiel Begleitgruppe Asse-II und Arbeitsgruppe Optionen-Rückholung

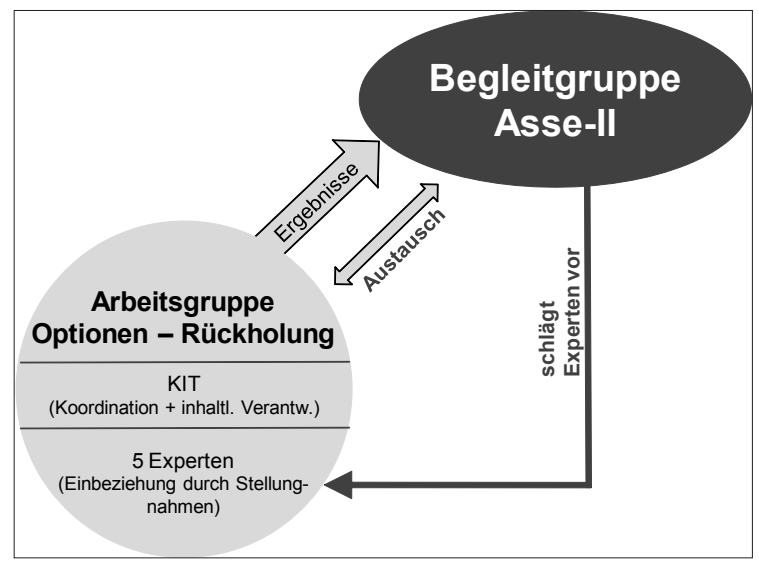

Quelle: BfS 
Seit Übernahme der Betreiberverantwortung wird das BfS an der AGO nur noch informell beteiligt. Die Federführung der AGO, an der mittlerweile fünf Experten der A2B beteiligt sind, liegt heute allein beim KIT. ${ }^{1}$ Der Bund unterstützt die Arbeiten durch Übernahme der Kosten für die Experten der A2B.

Sowohl die A2B als auch die AGO tagen regelmäßig und werden vom BfS über alle Planungsschritte und Arbeiten informiert. Prinzipiell läuft der Begleitprozess Asse in dieser Form bis heute. Die Vorschläge aus dem Gremium werden vom BfS nach fachlicher Prüfung bzw. Prüfung der Machbarkeit in die weiteren Planungen und Arbeiten einbezogen.

Die A2B wird vom BfS in Fachworkshops zu aktuellen Themen (z. B. Evaluierung und Beschleunigung der Rückholung) eingebunden.

\section{Wechsel des Betreibers}

Nach der Betriebsübernahme der Schachtanlage Asse II durch das BfS wurde ein neues Strahlenschutzregime eingerichtet und mit der Behebung vorher analysierter radiologischer und bergbaulicher Gefahrenquellen begonnen. Mit der Einholung der erforderlichen atom- und strahlenschutzrechtlichen Umgangsgenehmigungen wurden die atomrechtlichen Grundlagen für den Betrieb geschaffen. Für eine transparente und umfassende Öffentlichkeitsinformation wurden eine Info-Stelle vor Ort, ein Infomobil und eine Internetpräsentation (http://www.endlager-asse. de) eingerichtet, sowie umfangreiches Informationsmaterial (z. B. der Newsletter „Asse Einblicke") veröffentlicht. Zudem werden zu aktuellen Themen Infoveranstaltungen durchgeführt.

Im Verlaufe des Jahres 2009 erfolgte durch das BfS auf der Basis von Machbarkeitsstudien ein Vergleich verschiedener Optionen für die Stilllegung der Schachtanlage Asse II (Optionenvergleich). Bei diesem wurden die Vollverfüllung mit Verbleib der Abfälle am derzeitigen Ort, die interne Umlagerung der Abfälle und die Rückholung untersucht. Die Untersuchungen kamen zu dem Ergebnis, dass die Rückholung der Abfälle aus der Schachtanlage Asse II die beste Variante beim weiteren Umgang mit den dort eingelagerten radioaktiven Abfällen ist. Nach jetzigem Kennt- nisstand kann durch Rückholung und Verbringung in ein anderes Endlager die Langzeitsicherheit gewährleistet werden. Das Ergebnis wurde vom BfS am 15.1.2010 der Öffentlichkeit vorgestellt.

Zur Vorbereitung der Rückholung und Klärung offener Fragen wird eine Faktenerhebung (Probephase) durchgeführt. Dabei soll durch Anbohren, Öffnen und Bergen einzelner Abfallgebinde aus zwei ausgewählten Kammern die praktische Durchführbarkeit der Rückholung überprüft werden. Zurzeit wird in einem ersten Schritt die erste Kammer angebohrt, um mögliche explosive Gase, radioaktive Lösungen sowie den Zustand der Gebinde zu erkunden.

\section{Rahmenbedingungen und Erfordernisse für das Monitoring}

\subsection{Bergbaulicher Zustand und betriebliche Maßnahmen}

Mit der Schachtanlage Asse II hat das BfS die Betreiberverantwortung für ein Bergwerk übernommen, das ursprünglich zur Salzgewinnung eingerichtet und in das radioaktive Abfälle eingelagert wurden, ohne dass der für die Einrichtung eines Endlagers für radioaktive Abfälle erforderliche Nachweis der langzeitsicheren Verwahrung geführt wurde.

Durch den Salzabbau sind zahlreiche dicht beieinander liegende Abbaue in der Südflanke entstanden. Durch den Gebirgsdruck folgen Gebirgsbewegungen, die bereits zu einer Schädigung des Salzgesteins und des Nebengebirges geführt haben. Durch die Schädigungsprozesse sind Wegsamkeiten im Salzgestein und Deckgebirge entstanden. Durch diese tritt im oberen Teil des Baufeldes an der SW-Flanke in $500-575 \mathrm{~m}$ Tiefe Lösung aus dem Nebengebirge durch das aufgelockerte Salzgestein in die Grube ein. 1988 wurde erstmals festgestellt, dass salzgesättigtes Grundwasser aus dem Deckgebirge in das Grubengebäude eindringt (Zutrittslösung). Es wird ca. $12 \mathrm{~m}^{3} /$ Tag an Steinsalz $(\mathrm{NaCl})$ gesättigte Lösung aus dem Nebengebirge aufgefangen; an der Hauptauffangstelle auf der 658-m-Sohle sind es ca. 10,5 $\mathrm{m}^{3} /$ Tag. Da die Schädigungsprozesse andauern, besteht die Gefahr, dass der Lösungs- 
zutritt auf ein unbeherrschbares Niveau ansteigt und die Schachtanlage ,absäuft“.

Grundwasserzutritte sind im kommerziellen Salzbergbau nur aus Bergwerken bekannt, deren Abbauhohlräume keinen ausreichenden Sicherheitsabstand zum wasserführenden Deck- oder Nebengebirge aufweisen. Es existiert bislang kein Beispiel für eine erfolgreiche, dauerhafte Abdichtung eines solchen Lösungszutrittes. Im Zeitraum von 1995-2004 sind zur Stabilisierung der Schachtanlage vom ehemaligen Betreiber die Abbauhohlräume an der Südflanke mit 2,2 Mio. t Blasversatz aus feinkörnigem Steinsalz verfüllt worden. Um diesen an Porenvolumen reichen Versatz zu ertüchtigen, werden derzeit die Resthohlräume, die durch Sackung oben in den Kammern entstanden sind, mit Beton verfüllt (Firstspaltverfüllung). Dadurch soll eine Verlangsamung der Gebirgsbewegung erreicht werden, wodurch sich die Gefahr eines Anstieges des Lösungszutritts reduziert.

\subsection{Abgabepfade radioaktiver Stoffe in die Umwelt}

Die Verpackungen der radioaktiven Abfälle (in der Regel Rollreifenfässer, Rollsickenfässer und Blechtrommeln aus Stahl) dienten primär dem sicheren Antransport der Abfälle über Schiene und Straße und waren nicht als technisch (gas-) dichte Barriere konzipiert.

Messungen der Aktivitätskonzentration radioaktiver Stoffe in der Grubenluft (Wetter) belegen, dass aus den eingelagerten radioaktiven Abfälle flüchtige radioaktive Stoffe entweichen (hauptsächlich $\mathrm{HTO}^{2}, \mathrm{C}-14$-Verbindungen, Rn-222 und dessen Zerfallsprodukte) und über Wegsamkeiten aus den verschlossenen und zum Teil versetzten Einlagerungskammern in die mit Grubenluft versorgten Grubenbereiche gelangen sowie mit der abgeführten Grubenluft in die Umgebung abgeleitet werden.

Um das sukzessive Volllaufen des Bergwerks zu verhindern, werden monatlich ca. 300 $\mathrm{m}^{3}$ der aufgefangenen (gefassten) Zutrittslösung an das Bergwerk Maria Glück zur Verwertung abgegeben. Die restliche gefasste Zutrittslösung wird in der Schachtanlage Asse II verwertet oder soll, falls sie mit radioaktiven Stoffen kontaminiert ist, als radioaktiver Abfall entsorgt werden.

Über physikalisch-chemische Austauschprozesse kann ein Teil der aus den Abfällen entweichenden radioaktiven Stoffe, insbesondere HTO, über die Grubenluft in die Zutrittslösungen gelangen.

Sollte sich die Zutrittsrate erhöhen oder der Eintrittsort der Deckgebirgslösung zur Schachtanlage Asse II verlagern, könnte diese unter Umständen nicht mehr im erforderlichen Umfang gefasst werden und ein wesentlicher Teil die Auffangvorrichtungen umfließen. Die mögliche Folge wäre ein Kontakt mit den eingelagerten radioaktiven Abfällen. Mit dem Anstieg der Zutrittsrate und/oder einer Verlagerung des Zutrittsortes werden auch die Kapazitäten für die externe und interne Verwertung der Zutrittslösung zunehmend stärker ausgeschöpft, zumal die Wahrscheinlichkeit zunimmt, dass die Lösung mit radioaktiven Stoffe kontaminiert sein kann. Der Zustand, dass die Zutrittslösung nicht mehr sicher gefasst und einer Verwertung zugeführt werden kann, wird als ,auslegungsüberschreitender Lösungszutritt" betrachtet.

Die Wahrscheinlichkeit für das Szenario des auslegungsüberschreitenden Lösungszutritts ist nicht quantifizierbar. Aufgrund der Tatsache, dass der Salzstock bereits seine Integrität gegenüber den grundwasserführenden Schichten verloren hat, besteht aber unter Experten Einigkeit darüber, dass es sich um ein realistisches Szenario handelt. Im Rahmen eines vom BfS initiierten Fachgespräches am 24.10.2007 mit dem BMU und Experten aus Bergbau und Wissenschaft wurde dies als eine wesentliche Konsequenz der Verformungen des Tragsystems benannt.

Für den Fall, dass es zu dem auslegungsüberschreitenden Lösungszutritt in die Schachtanlage Asse II kommt, kann eine Freisetzung von Radionukliden über den Grundwasserpfad in die Biosphäre und möglicherweise in die Nahrungskette des Menschen und damit Auswirkungen für künftige Generationen nicht ausgeschlossen werden. Daher sind Maßnahmen zur Minimierung der Konsequenzen eines solchen Ereignisses erforderlich (Notfallvorsorge). Im Rahmen der Notfallvorsorge werden die Zugänge zu den Abfallkam- 
mern abgedichtet und die Hohlräume unter und im Niveau der Abfälle mit Beton verfüllt.

\subsection{Schutz der Bevölkerung und Umwelt}

Zum Schutz der Bevölkerung und der Umwelt regelt $\S 48$ der Strahlenschutzverordnung (StrlSchV), dass die Ableitung radioaktiver Stoffe aus der Schachtanlage Asse II in die Umgebung zu überwachen ist (StrlSchV 2011). Wie dabei vorzugehen ist, ist in der Richtlinie zur Emissions- und Immissionsüberwachung kerntechnischer Anlagen (REI) festgelegt (REI 2005). Weitere Vorgaben gehen aus dem Genehmigungsbescheid für die Schachtanlage Asse II über den Umgang mit radioaktiven Stoffen gemäß § 7 Strahlenschutzverordnung hervor (NMU 2010). Die mit der Grubenluft in die Umgebung abgeleiteten radioaktiven Stoffe werden an dem Punkt, an dem die Grubenluft aus der Schachtanlage geleitet wird (am Abwetterbauwerk), messtechnisch erfasst (Emissionsüberwachung). Die Messprogramme zur Emissionsüberwachung und zur Umgebungsüberwachung (Immissionsüberwachung) sind im Kapitel 4.4 dargestellt.

Gemäß den $\S \S 46$ und 47 der StrlSchV darf die Summe der Strahlenexpositionen aus der Direktstrahlung und der Strahlenexposition aus Ableitungen für Einzelpersonen der Bevölkerung nicht höher als 1 Millisievert im Kalenderjahr sein.

Vor der Abgabe der Zutrittslösung zur Verwertung im Bergwerk Maria Glück wird gemessen, wie viel Tritium diese enthält. Die gemessene Aktivitätskonzentration liegt dabei mehrere GröBenordnungen unter den Werten, für die durch Modellberechnungen nachgewiesen wurde, dass das Schutzziel der StrlSchV für die Freigabe radioaktiver Stoffe aus der strahlenschutzrechtlichen Überwachung ( $§ 29 \mathrm{StrlSchV}$ ) eingehalten wird. Die Freigabe darf nur erfolgen, wenn sichergestellt ist, dass hierbei für Einzelpersonen der Bevölkerung die effektive Dosis im Bereich von 10 Mikrosievert im Kalenderjahr auftreten kann.

Um die möglichen radiologischen Konsequenzen für Mensch und Umwelt infolge eines auslegungsüberschreitenden Lösungszutritts und der dadurch notwendigen Aufgabe des Offenhaltungsbetriebes der Schachtanlage Asse II bewerten zu können, müssen diese zunächst belastbar und dem Stand von Wissenschaft und Technik entsprechend ermittelt werden. Hierzu sind Modellrechnungen durchzuführen, die die Mobilisierung und Freisetzung der Radionuklide aus den Abfällen, deren Transport im Grubengebäude, durch das Deckgebirge und mit dem Grundwasser umfassen. In die Modellrechnungen fließt eine Vielzahl von Modellparametern ein. Außerdem sind die stattfindenden Prozesse detailliert abzubilden. Es sind zeitintensive Untersuchungen und Erhebungen erforderlich, um ein belastbares Ergebnis zu erzielen. Viele dieser Modellparameter und -prozesse sind noch unbekannt und wurden durch Annahmen ersetzt bzw. durch Parametervariationen berücksichtigt, um erste Abschätzungen darüber zu ermöglichen, in welcher Bandbreite die potenzielle Strahlenexposition für die Bevölkerung liegen könnte. Nach derzeitigen Kenntnisstand und unter Berücksichtigung der Modellunsicherheiten sowie der Abhängigkeit von der anzuwendenden Konservativität bei der Expositionsermittlung ist nicht auszuschließen, dass die Einhaltung von Schutzzielen, wie sie in einem Genehmigungsverfahren nachzuweisen wären, nicht gewährleistet werden können. Die Frage, in welchem Maß die potenziellen Konsequenzen bei Eintritt des Szenarios durch die verbleibenden Handlungsoptionen verringert werden können und mit welchem Gesundheitsrisiko die unvermeidbaren Belastungen verbunden sind, ist noch zu beantworten.

Die Ergebnisse der vorliegenden Abschätzung waren für das BfS Anlass, Vorsorge- und Notfallmaßnahmen zu planen und als Gefahrenabwehrmaßnahme umzusetzen. Sie verfolgen das Ziel, die möglichen negativen Konsequenzen soweit wie möglich zu minimieren.

\subsection{Messprogramme zur Emissions- und Immissionsüberwachung}

Zur Beurteilung der Lage sind für die ausreichende Vorsorge und entsprechend auf für potenziell notwendige Gefahrenabwehr sowohl Messprogramme zur Überwachung von Emissionen und Immissionen bedeutsam.

Zur Emissionsüberwachung ist an dem Punkt, an dem die Grubenluft aus der Schachtanlage geleitet wird (Abwetterbauwerk), eine 


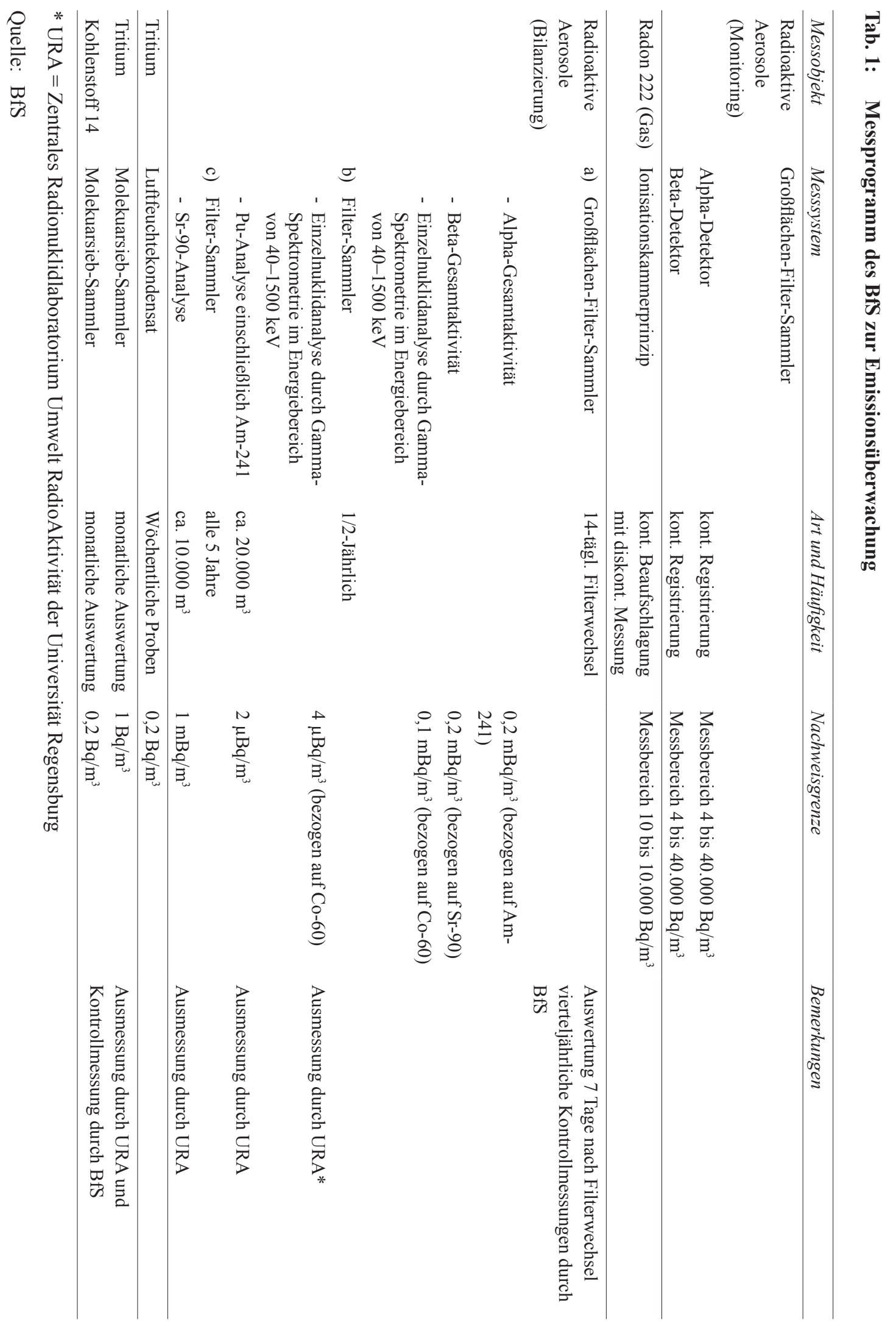


Probeentnahmeeinrichtung installiert. Die Immissionsüberwachung erfolgt über ein engmaschiges Kontrollnetz in der Umgebung der Schachtanlage Asse. ${ }^{3}$

\subsubsection{Emissionsüberwachung}

Die Ableitungen radioaktiver Stoffe mit der abgeführten Grubenluft (Emissionen) aus der Schachtanlage Asse II werden durch das BfS als Genehmigungsinhaber gemäß folgendem Messprogramm überwacht. Dabei wird kurz- und langlebigen Aerosolen sowie Tritium und C14 besondere Aufmerksamkeit geschenkt (s. Tab.1).

\subsubsection{Immissionsüberwachung}

Nach REI (2005) sind für die Umgebungsüberwachung („Immissionsüberwachung“) zwei Messprogramme durchzuführen:

- ein Programm, das vom Genehmigungsinhaber BfS durchzuführen ist und

- ein ergänzendes und kontrollierendes Programm, das von einer unabhängigen Messstelle durchzuführen ist.

Das Messprogramm des BfS ist auf die drei Umweltbereiche Luft, Boden und Wasser ausgerichtet und ist in den Tabellen 2, 3 und 4 dargestellt.

Ergänzend $\mathrm{zu}$ den gesetzlich geforderten Überwachungsmaßnahmen erfolgt als vertrauensbildende Maßnahme eine zusätzliche Umgebungsüberwachung durch die „LUFA-ITL $\mathrm{GmbH}^{\prime}$. Die Ergebnisse der LUFA werden in Jahresberichten veröffentlicht, die auf der Internetseite der Infostelle Asse veröffentlicht sind (http://www.endlager-asse.de).

Um den bei der Bevölkerung vorhandenen Ängsten zu begegnen, hat das BfS nach der Übernahme der Betreiberverantwortung unmittelbar an der Schachtanlage Asse II oberirdisch eine Gamma-Ortsdosisleistung (ODL-) Messsonde installiert, wie sie ansonsten auf der Basis des Strahlenschutzvorsorgegesetzes vom BfS zur Überwachung der Umweltradioaktivität betrieben werden. Auf ausdrücklichen Wunsch der Bevölkerung wurden zudem rund um die Schachtanlage Asse II sechs weitere Messsonden aufgestellt. Dies geschah, obwohl ein messtechnischer Nachweis von radioaktiven Stoffen aus der Schachtanlage Asse II in der Umwelt nicht zu erwarten ist (s. folgendes Unterkapitel).

Außerdem führte die Leitstelle Trinkwasser des BfS in Berlin auf Bitte der Gemeindeverwaltung monatliche Untersuchungen des Trinkwassers der Gemeinde Kissenbrück auf H-3 und Cs-137 durch, solange diese über eine Wasserversorgung aus lokalen Brunnen verfügte. Dies erfolgte als vertrauensbildende Maßnahme, obwohl auch hier eine Belastung fachlich ausgeschlossen werden kann. Mittlerweile erfolgt die Trinkwasserversorgung der Gemeinde über eine Fernwasserleitung.

\subsection{Bewertung der Messergebnisse und Dokumentation}

Der Genehmigungsinhaber BfS und die unabhängige Messstelle haben ihre Messergebnisse aus der Emissions- und Immissionsüberwachung aufzuzeichnen und in Quartals- und Jahresberichten darzustellen und der zuständigen Behörde vorzulegen.

Zusätzlich werden die Ergebnisse in einem Jahresbericht „Strahlenschutz und Umgebungsüberwachung im Bereich der Schachtanlage Asse II" dargestellt (BfS 2012).

Die mit der Fortluft der Schachtanlage Asse II abgeleiteten Radionuklide führen zu keiner messtechnisch erfassbaren Erhöhung der Radionuklidkonzentration in der Umgebung. Auch an den ODL-Messsonden wurden keine auffälligen Abweichungen vom normalen Strahlungspegel festgestellt.

Die potenzielle Strahlenexposition der Bevölkerung wird den gesetzlichen Vorgaben entsprechend unter Verwendung von konservativen Rechenmodellen aus den gemessenen Ableitungen berechnet, um die Unterschreitung der Dosisgrenzwerte der StrlSchV belegen zu können. Für das Jahr 2011 z. B. zeigen diese Berechnungen, dass die maximale potenzielle Dosis für die kritische Altergruppe (Säugling < $1 \mathrm{Jahr}$ ) mit 32 $\mu \mathrm{Sv} / \mathrm{Jahr}$ deutlich unter dem zulässigen Grenzwertes gemäß $§ 47 \mathrm{StrlSchV} \mathrm{liegt.} \mathrm{Für} \mathrm{alle} \mathrm{ande-}$ ren Altersgruppen sind die errechneten Werte für die potenzielle Strahlenexposition noch geringer. 


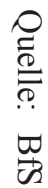

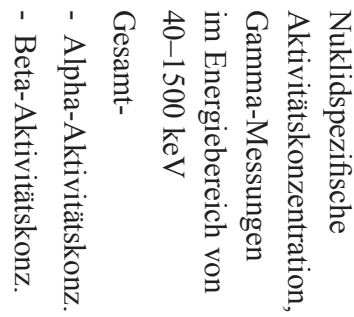

in

恳署

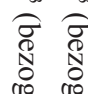

$\begin{array}{cc}0 \\ 0 \\ 9 \\ 9 & 9 \\ 9 & 9 \\ 9 & 9\end{array}$

节 若

急

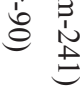

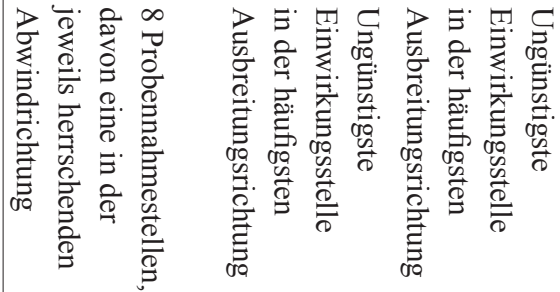

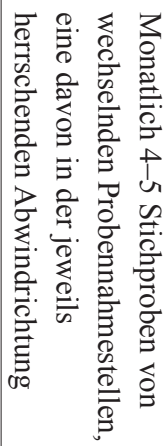

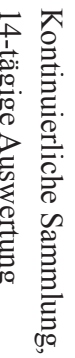

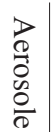

ㅇ $\overline{8} \overline{8}$

छ్

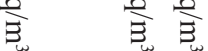

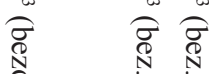

व

焉

变

高

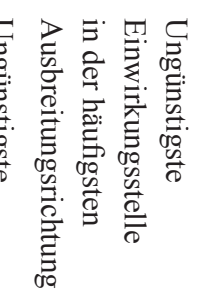

言

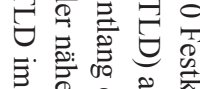

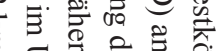

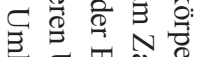

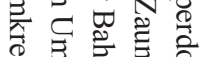

ज.

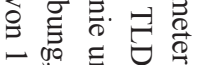

空言

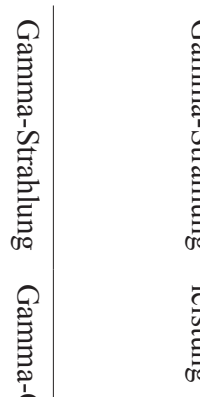

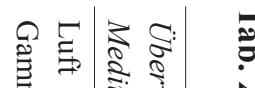

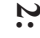

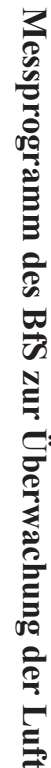

离:

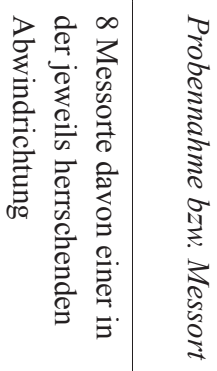

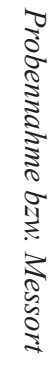

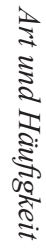

政票

可

产

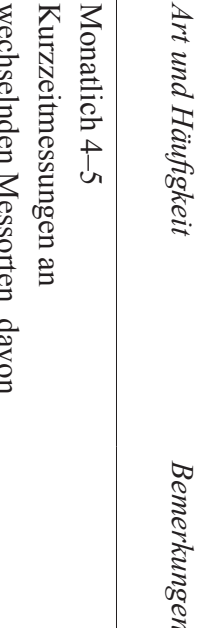

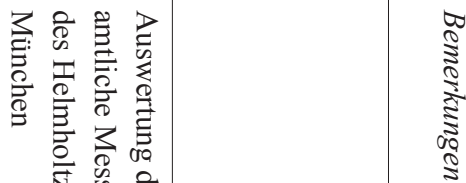

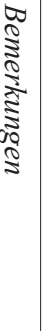


Tab. 3: Messprogramm des BfS zur Überwachung des Bodens

\begin{tabular}{|c|c|c|c|c|}
\hline $\begin{array}{l}\text { Überwach- } \\
\text { tes Medium }\end{array}$ & Messgröße & Nachweisgrenze & Probennahme bzw. Messort & Art und Häufigkeit \\
\hline \multirow[t]{2}{*}{ Boden } & $\begin{array}{l}\text { Gammaspektrometrie im Ener- } \\
\text { giebereich von } 40-1500 \mathrm{keV} \text {, } \\
\text { spezifische Aktivität einzelner } \\
\text { Radionuklide }\end{array}$ & $\begin{array}{l}0,5 \mathrm{~Bq} / \mathrm{kg} \text { bezo- } \\
\text { gen auf Co- } 60 \\
\text { und Trocken- } \\
\text { masse }\end{array}$ & $\begin{array}{l}4 \text { Probennahmestellen davon } \\
\text { eine Referenzstelle }\end{array}$ & $\begin{array}{l}\text { jeweils } 2 \text { Stichpro- } \\
\text { ben pro Jahr }\end{array}$ \\
\hline & $\begin{array}{l}\text { Gesamt-Beta-Aktivitätsflächen- } \\
\text { belegung }\end{array}$ & $4 \mathrm{kBq} / \mathrm{m}^{2}$ & $\begin{array}{l}3 \text { Messorte in der Nähe des } \\
\text { Zauns, zusätzlich einer in der } \\
\text { jeweils herrschenden Abwind- } \\
\text { richtung }\end{array}$ & $\begin{array}{l}\text { jeweils } 2 \text { Kurz- } \\
\text { zeitmessungen pro } \\
\text { Jahr }\end{array}$ \\
\hline Gras & $\begin{array}{l}\text { Gammaspektrometrie im Ener- } \\
\text { giebereich von } 40-1500 \mathrm{keV} \text {, } \\
\text { spezifische Aktivität einzelner } \\
\text { Radionuklide }\end{array}$ & $\begin{array}{l}0,5 \mathrm{~Bq} / \mathrm{kg} \text { bezo- } \\
\text { gen auf Co- } 60 \\
\text { und Feuchtmasse }\end{array}$ & $\begin{array}{l}4 \text { Probennahmestellen davon } \\
\text { eine Referenzstelle }\end{array}$ & $\begin{array}{l}\text { jeweils } 2 \text { Stichpro- } \\
\text { ben pro Jahr }\end{array}$ \\
\hline
\end{tabular}

Quelle: BfS

Tab. 4: Messprogramm des BfS zur Überwachung des Wassers

\begin{tabular}{|c|c|c|c|c|c|}
\hline $\begin{array}{l}\text { Überwach- } \\
\text { tes Medium }\end{array}$ & Messgröße & Nachweisgrenze & $\begin{array}{l}\text { Probennahme bzw. } \\
\text { Messort }\end{array}$ & Art und Häufigkeit & Bemerkung \\
\hline $\begin{array}{l}\text { Grund- und } \\
\text { Oberflä- } \\
\text { chenwasser }\end{array}$ & $\begin{array}{l}\text { Gesamt-Beta-Aktivi- } \\
\text { tätskonzentration }\end{array}$ & $\begin{array}{l}0,2 \mathrm{~Bq} / 1 \text { bezogen } \\
\text { auf } \mathrm{K}-40\end{array}$ & $\begin{array}{l}19 \text { Probennahmestellen } \\
\text { an Quelle, Brunnen und } \\
\text { Vorflutern }\end{array}$ & $\begin{array}{l}\text { jeweils } 1 / 4 \text { jährli- } \\
\text { che Stichproben }\end{array}$ & $\begin{array}{l}\text { Bei Werten } \\
\text { über } 0,2 \mathrm{~Bq} / 1 \\
\text { erfolgt die } \\
\text { Bestimmung } \\
\text { der Rest-Be- } \\
\text { ta-Aktivitäts- } \\
\text { konzentration }\end{array}$ \\
\hline
\end{tabular}

Quelle: BfS

Dennoch erfolgen als vertrauensbildende Maßnahmen ergänzende Untersuchungen (Futtermittel, Grundwasser) und zusätzliche ODL-Messungen in der Umgebung der Schachtanlage Asse, die über die gesetzlichen und fachlichen Erfordernisse hinausgehen.

\subsection{Veröffentlichung der Messwerte und Ergebnisse}

Auf der Internetseite der Infostelle Asse kann sich jede Bürgerin und jeder Bürger über die Messwerte der ODL-Messsonden informieren (http://www.endlager-asse.de). Die Jahresberichte ,Strahlenschutz und Umgebungsüberwachung im Bereich der Schachtanlage Asse II“" der Asse
GmbH, die Quartals- und Jahresberichte der Asse GmbH zur Emissions- und Immissionsüberwachung gemäß REI (2005) und der unabhängigen Messstelle sowie die Berichte der LUFA über die zusätzliche Umgebungsüberwachung sind dort neben vielfältigen Informationen zu der Schachtanlage Asse II (z. B. zum Gesundheitsmonitoring des BfS, das für ehemalige Asse-Mitarbeiter durchgeführt wird) ebenfalls einsehbar.

\section{Zusammenfassung}

Der derzeit laufende Entscheidungsprozess zur Stilllegung der Schachtanlage Asse II und die Planung für die Rückholung der Abfälle wird von der Asse-II-Begleitgruppe des Landkreises 
Wolfenbüttel aus örtlichen Stakeholdern und Bürgerinitiativen begleitet. Diese unterbreitet auch Vorschläge für ein Umweltmonitoring.

Die aus der Schachtanlage Asse II mit der abgeführten Grubenluft in die Umgebung abgeleiteten radioaktiven Stoffe werden gemäß den gesetzlichen Vorgaben messtechnisch überwacht. Auch wird ein Umgebungsüberwachungsprogramm durchgeführt. Berechnungen zeigen, dass die maximale potenzielle Strahlenexposition deutlich unter den zulässigen Grenzwerten der Strahlenschutzverordnung liegt. Als vertrauensbildende Maßnahmen erfolgen ergänzende Untersuchungen (Futtermittel, Grundwasser) und zusätzliche ODL-Messungen in der Umgebung der Schachtanlage Asse II, die über die gesetzlichen und fachlichen Erfordernisse hinausgehen.

Eine transparente und umfassende Information der Öffentlichkeit wird insbesondere über eine Info-Stelle („Info Asse“), ein Infomobil, eine Internetpräsentation und Info-Broschüren gewährleistet. Darüber hinaus werden zu aktuellen Themen Infoveranstaltungen durchgeführt.

\section{Anmerkungen}

1) Nähere Informationen zur AGO und zum Begleitprozess Asse sind im Internet auf der Homepage der Begleitgruppe Asse-II unter http://www.asse2-begleitgruppe.de/index.php?id=44 abrufbar.

2) HTO ist Tritium-(H-3-)haltiges Wasser.

3) Siehe BfS 2011; Asse Einblicke Nr. 12; Download unter http://www.endlager-asse.de/SharedDocs/ Publikationen.

\section{Literatur}

AtG - Atomgesetz, 2011: Gesetz über friedliche Verwendung der Kernenergie und Schutz gegen ihre Gefahren (Atomgesetz) in der Fassung der Bekanntmachung vom 15. Juli 1985 (BGBI. I S. 1565) zuletzt geändert durch Art. $4 \mathrm{G}$ über die Neuordnung des Geräte- und Produktsicherheitsrechts vom 8 . November 2011 (BgBI. I S. 2178)

BfS - Bundesamt für Strahlenschutz, 2011: Asse Einblicke Nr 12 - Informationsschrift zum Endlager Asse II, Wie die Asse und die Region überwacht werden. Salzgitter, März 2011; http://www.endlager-asse.de/ SharedDocs/Publikationen

BfS - Bundesamt für Strahlenschutz, 2012: Strahlenschutz und Umgebungsüberwachung im Bereich der
Schachtanlage Asse II - Jahresbericht 2011, Stand 06.09.2012; http://www.endlager-asse.de/SharedDocs/Downloads/DE/Umgebungsueberwachung

NMU - Niedersächsisches Ministerium für Umwelt und Klimaschutz, 2010: Genehmigungsbescheid für die Schachtanlage Asse II, Bescheid 1/2010, Umgang mit radioaktiven Stoffen gemäß § 7 Strahlenschutzverordnung (StrlSchV), Hannover, 08.07.2010;http:// www.endlager-asse.de/SharedDocs/Downloads/DE/ Genehmigungsunterlagen/

REI, 2005: Richtlinie zur Emissions- und Immissionsüberwachung kerntechnischer Anlagen vom 7. Dezember 2005 (GMB1. 2006, Nr. 14-17, S. 254)

Schauermann, V., 2007: Abschlussbetriebsplan für die Schachtanlage Asse II. Rev. 00. Remlingen, 26.01.2007 (nicht beschieden, unveröffentlicht)

StrlSchV - Strahlenschutzverordnung, 2011: Verordnung über den Schutz vor Schäden durch ionisierende Strahlen: Vom 20. Juli 2001 (BGBI. I. S. 1714, ber. BGBI. 2002 I S. 1459) zuletzt geändert durch Art. 1 VO zur Änd. Strahlenschutzrechtlicher VO vom 4.10.2011 (BGBI I S. 2000)

\section{Kontakt}

Dr. Christiane Wittwer

Bundesamt für Strahlenschutz

Willy-Brandt-Straße 5, 38226 Salzgitter

Tel.: +49 (0) $30 / 18333$ - 1936

E-Mail: cwittwer@bfs.de

$《 》$ 